\title{
Evaluation of Multiaxial Creep-fatigue Strength for High Chromium Steel under Non-proportional Loading
}

\author{
Yuuki Kasamuta ${ }^{1}$, Fumio Ogawa ${ }^{2}$, Takamoto Itoh $^{2,2}$, and Hiroyasu Tanigawa ${ }^{3}$ \\ ${ }^{1}$ Graduate School of Science and Engineering, Ritsumeikan University, Japan \\ ${ }^{2}$ College of Science and Engineering, Ritsumeikan University, Japan \\ ${ }^{3}$ National Institutes for Quantum and Radiological Science and Technology, Japan
}

\begin{abstract}
This study discusses the result of creep-fatigue tests of a highchromium steel, $\mathrm{F} 82 \mathrm{H}$ which was designed as blanket structural materials of nuclear fusion reactor, carried out at room temperature to $823 \mathrm{~K}$ in air. Strain paths applied were a push-pull loading and a circle loading in which normal and shear strain have 90 degree phase difference. The holding times used were $180 \mathrm{~s}$ and $600 \mathrm{~s}$. Moreover, an evaluation of failure life by taking into account intensities of creep and non-proportionality is discussed based on both the life evaluation proposed by Itoh, et al and method of modified universal slopes. Availability of the equation for the life evaluation was confirmed by comparison with conventional universal slope method.
\end{abstract}

\section{Introduction}

Nuclear fusion reactor is used as a kind of large scale power generation around the world ${ }^{1)}$, and diversified researches are performed toward implementation of the power generation by nuclear fusion reactor. As one of the problems, reduction in fatigue life of used materials under high temperature is pointed out. On the other hand, high chromium steel, F82H steel, was applied as a first wall of nuclear fusion reactor. This material is similar to Mod.9Cr-1Mo steel which was used as fast breeder reactor ${ }^{2}$ (FBR). The difference of two materials is that Mo is replaced to $\mathrm{W}$ leading to increased high temperature strength, and $\mathrm{Nb}$ is replaced to improve characteristics for low induced radioactivity. The materials used under high temperature environment has been subjected to the thermal loading in addition to the mechanical loading resulting in the non-proportional loading in which principal directions of stress and strain are changed in a cycle. As a result of this case, the failure life of materials dramatically decreases compared with proportional loading.

In this study, creep-fatigue tests of high chromium steel at ambient and high temperatures under non-proportional loading were conducted. Moreover, those data were evaluated by means of both the parameter concerning creep damage and non-proportionality proposed by Itoh, et al and method of modified universal slopes. Moreover, whether the equation has more validity in comparison with usual universal slope method was discussed to investigate the suitability of the modified equation as a design standard under non-proportional loading.

\footnotetext{
${ }^{2}$ Corresponding author : itohtaka@ fc.ritsumei.ac.jp
} 


\section{Test condition}

\subsection{Testing machine}

Figure 1 shows the testing machine used in this study. The electrical servo-controlled tensiontorsion fatigue testing machine which can combine push-pull loading and torsional loading was used. This testing machine is equipped with two types of the cylinder for axial loading and torsion loading, and it enabled fatigue tests under non-proportional loading in which axial and torsional strain have 90-degree phase difference. Figure 2 shows strain paths and waveforms applied.

The specifications of the machine is that maximum axial loading and maximum torque are $50 \mathrm{kN}$ and $500 \mathrm{Nm}$, respectively. As a measurement method, loads were measured by the load cell, and each strain was measured by the extensometer equipped with the eddy current sensor. In this machine, the induction heating apparatus was used to conduct the creep-fatigue test under high temperature.

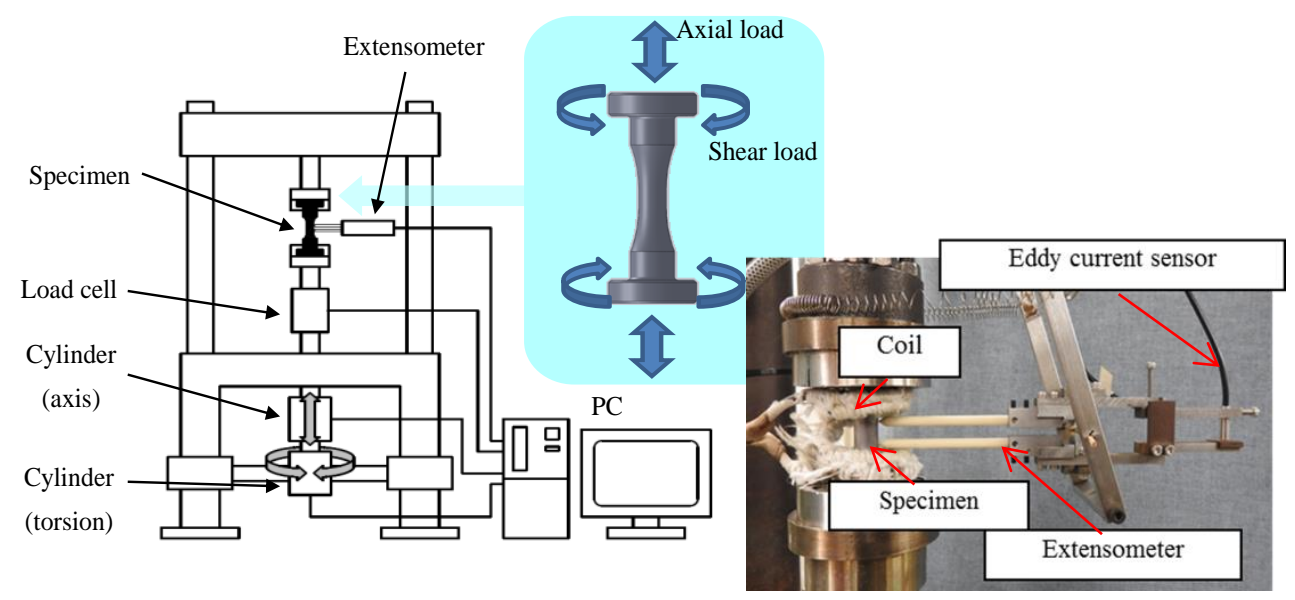

Fig.1. Tension-torsion fatigue testing machine and extensometer used.

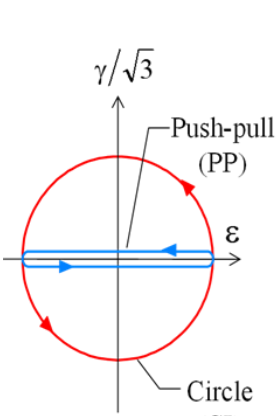

(CI)
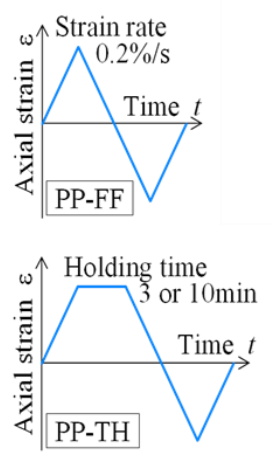

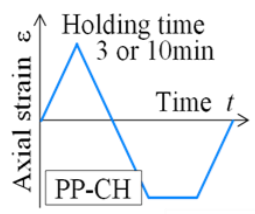

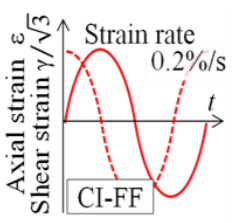

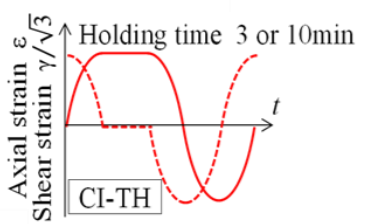

Fig. 2. Strain paths and waveforms. 


\subsection{Test specimen}

Figure 3 shows shape and dimension of the specimen for creep-fatigue test used. Material used were high chromium steel, F82H steel using a hollow cylinder specimen to apply axial stress and shear stress in the plane stress state. The outer diameter of $12 \mathrm{~mm}$ inter diameter of $9 \mathrm{~mm}$, and parallel part length of $8 \mathrm{~mm}$ were adopted.

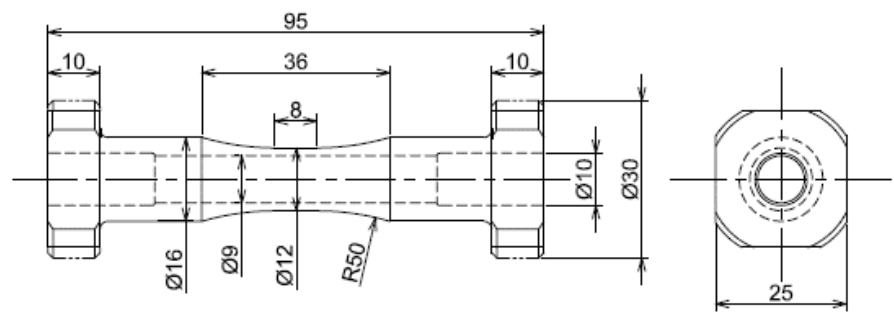

Fig. 3. A hollow cylinder specimen. (mm)

\subsection{Test condition}

\subsubsection{Fatigue test under push-pull loading}

Fatigue test under push-pull loading were conducted at room temperature, $573 \mathrm{~K}$ in air, and $823 \mathrm{~K}$ in air. Moreover, data reduction of those data was performed by means of the method of modified universal slopes. A total strain range in the method of modified universal slopes can be defined as,

$$
\Delta \varepsilon=0.0266 \varepsilon_{\mathrm{f}}^{0.155}\left(\frac{\sigma_{\mathrm{B}}}{E}\right)^{-0.53} N_{\mathrm{f}}^{-0.56}+1.17\left(\frac{\sigma_{\mathrm{B}}}{E}\right)^{0.832} N_{\mathrm{f}}^{-0.09}
$$

where $\sigma_{\mathrm{B}}, E$, and $\varepsilon_{\mathrm{f}}$ are the tensile strength, Young's modulus, and fracture strength, respectively. $\sigma_{\mathrm{B}}$ was defined a figure in Ref. (3). $E^{4)}$ can be defined as,

$$
\mathrm{E}=218.76-0.077834 \mathrm{~T}+1.4735 \times 10^{-4} \mathrm{~T}^{2}-2.1998 \times 10^{-7} \mathrm{~T}^{3}
$$

where $\mathrm{T}$ is the testing temperature. $\varepsilon_{\mathrm{f}}$ was defined by the data fitting after substituting both $\sigma_{\mathrm{B}}$ and $E$. Fatigue test at $823 \mathrm{~K}$ in air were conducted using a solid round bar specimen.

\subsubsection{Creep-fatigue test under non-proportional loading}

An analysis for the creep-fatigue test data under non-proportional loading was conducted. Creep-fatigue test taking account of the non-proportionality were conducted at $823 \mathrm{~K}$ in air. Total axial strain and total shear strain ranges were the same when they are correlated using von Mises strain. A strain range in the method of modified universal slopes taking into account strain paths and creep damage can be defined as,

$$
\Delta \varepsilon=\frac{0.0266 \varepsilon_{\mathrm{f}}^{0.155}\left(\frac{\sigma_{\mathrm{B}}}{E}\right)^{-0.53}}{\left(1+\alpha K_{\mathrm{S}} f_{\mathrm{NP}}\right) K_{\mathrm{V}}} N_{\mathrm{f}}^{-0.56}+1.17\left(\frac{\sigma_{\mathrm{B}}}{E}\right)^{0.832} N_{\mathrm{f}}^{-0.09}
$$


where $f_{\mathrm{NP}}$ is a non-proportional factor, the parameter expressing the severity of nonproportional straining. Figure 4 shows definitions of principal strain range and mean principal value. $f_{\mathrm{NP}}$ is defined as,

$$
f_{\mathrm{NP}}=\frac{\pi}{2 S_{\text {Imax }} L_{\text {path }}} \int_{\mathrm{C}}\left|e_{1} \times e_{\mathrm{R}}\right| S_{\mathrm{I}}(\mathrm{t}) \mathrm{ds}, L_{\text {path }}=\int_{\mathrm{C}} \mathrm{ds}
$$

where $S_{\mathrm{I}}(t)$ is the maximum absolute values of principal strain at the time. $S \mathrm{I}_{\max }$ is the maximum value of $S_{\mathrm{I}}(t)$ under the strain path. $e_{1}$ and $e_{\mathrm{R}}$ are the unit vectors of $S_{\mathrm{I}}\left(\mathrm{t}_{0}\right)$ direction and $S_{\mathrm{I}}(\mathrm{t})$ direction, respectively. ds is the increment of strain path. $C$ is integration route of strain paths. " $\times$ " shows vector product. $L_{\text {path }}$ is the sum of total strain paths. For strain path under proportional loading and circle loading, $f_{\mathrm{NP}}$ takes 0 and 1 , respectively.

$\alpha$ is a material constant, the parameter related to the additional hardening due to nonproportional straining. Moreover, this parameter can be defined by 3 ways, (a) deciding from a degree of additional hardening, (b) fitting the data under circle loading to that under pushpull loading, and (c) calculating from the result of tensile test proposed by the authors. In this paper, $\alpha$ was decided by (a). $\alpha^{5}$ can be defined as,

$$
\alpha=S \frac{\sigma_{\mathrm{B}}-\sigma_{\mathrm{Y}}}{\sigma_{\mathrm{B}}}
$$

where $S$ is a material constant considering the difference of the crystal structure. $\sigma_{Y}$ is a yielding stress or $0.2 \%$ proof strength. $\sigma_{\mathrm{B}}, E$ and $\varepsilon_{\mathrm{f}}$ were taken as the same values as the result of 2.3.1.

Moreover, $K_{\mathrm{s}}$ and $K_{\mathrm{v}}$ are the parameter considering strain rate and the parameter considering the decrease of non-proportionality caused by relaxation, respectively. $K_{\mathrm{s}}$ and $K_{\mathrm{v}}$ are indicated by,

$$
K_{\mathrm{s}}=\chi^{\left(\frac{v_{2}}{v_{1}}-1\right)}, K_{\mathrm{v}}=\left(\frac{v_{2}}{v_{1}}\right)^{(\kappa-1) \beta}
$$

where $\chi, \beta$ and $\kappa$ are material constants which can be calculated by the result of fatigue data under push-pull loading and circle loading. $v_{1}$ is the frequencies corresponding to the strain rate of the fatigue test in which creep effect can be ignored. $v_{2}$ is the frequencies with the lower strain rate in which creep effect is included. The time of holding were 3 minutes (180 s) and 10 minutes $(600 \mathrm{~s})$, and tension holding and compression holding were indicated by ' $\mathrm{TH}$ ' and ' $\mathrm{CH}$ ', respectively.
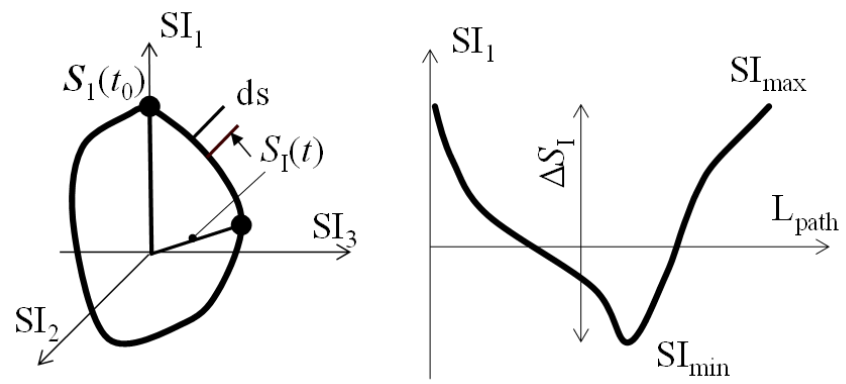

Fig. 4. Definitions of principal strain range and mean pricipal value. 


\section{Analytical result}

\subsection{Fatigue test results under push-pull loading}

Table 1 shows each value of $\sigma_{\mathrm{B}}, E$ and $\varepsilon_{\mathrm{f}}$. Figure 5 shows the result of fatigue test under pushpull loading and fatigue life curve based on the method of modified universal slopes. Because number of cycle to failure of fatigue test using a hollow cylinder specimen is a half of the result using a solid round bar specimen, number of cycle to failure of fatigue test using a hollow cylinder specimen was evaluated with the value of twice as large as the value obtained by fatigue test for solid round bar specimen.

As you can see, it can be confirmed from Table 1 that fracture strains are similar values under all the test temperatures. Moreover, fracture strain obtained by the data fitting was evaluated properly to indicate the similar value to the result of tensile test. Moreover, the result of fatigue test under all the test temperature can be also evaluated with the similar curves by means of the method of modified universal slopes. Therefore, regardless of different test temperatures, the fatigue data of F82H steel can be evaluated by the method of modified universal slopes to $823 \mathrm{~K}$ in air, and it is confirmed that fatigue life of F82H steel did not largely decrease. It means that the result of fatigue test can be predicted by using the result of static tensile test; the fatigue data can be evaluated without fatigue test.

Table 1. Properties of F82H steel under each test.

\begin{tabular}{|c|c|c|c|}
\hline $\begin{array}{c}\text { Test temperature } \\
(\mathbf{K})\end{array}$ & $\begin{array}{c}\text { Tensile strength } \\
(\mathbf{M P a})\end{array}$ & $\begin{array}{c}\text { Young's modules } \\
(\mathbf{G P a})\end{array}$ & Elongation \\
\hline 300 & 620 & 217 & 0.20 \\
\hline 573 & 553 & 203 & 0.18 \\
\hline 823 & 420 & 184 & 0.22 \\
\hline
\end{tabular}

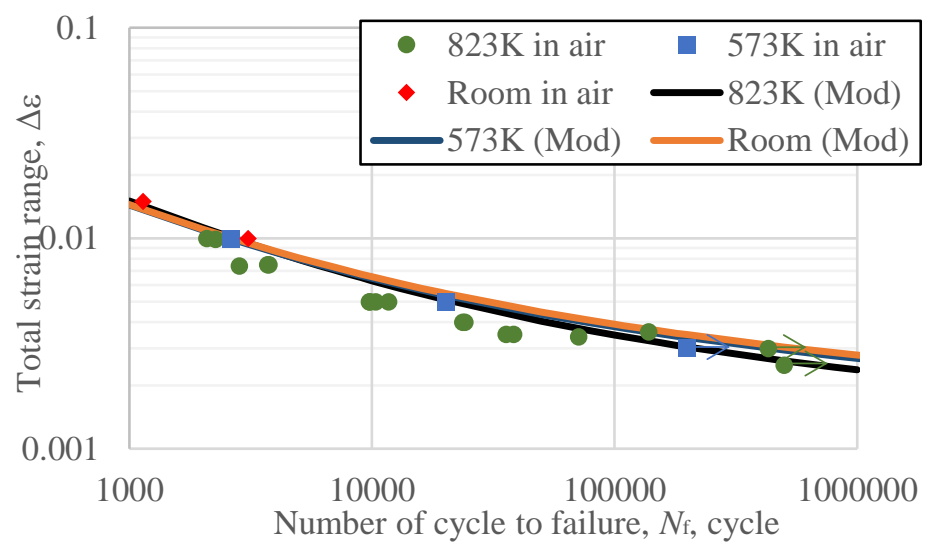

Fig. 5. Correlated $N_{\mathrm{f}}$ with total strain range. 


\subsection{Creep-fatigue test results under non-proportional loading}

Figure 6 shows the correlated $N_{\mathrm{f}}$ using Eq. (3). The solid line and the plot line on the figure were defined similarly to those in section 3.2. As can be seen, the results of creep-fatigue data were correlated within the factor of 2 band by using Eq. (3). Therefore, by considering non-proportionality and creep damage in $\mathrm{F} 82 \mathrm{H}$ steel, all the data can be evaluated by means of Eq. (3).

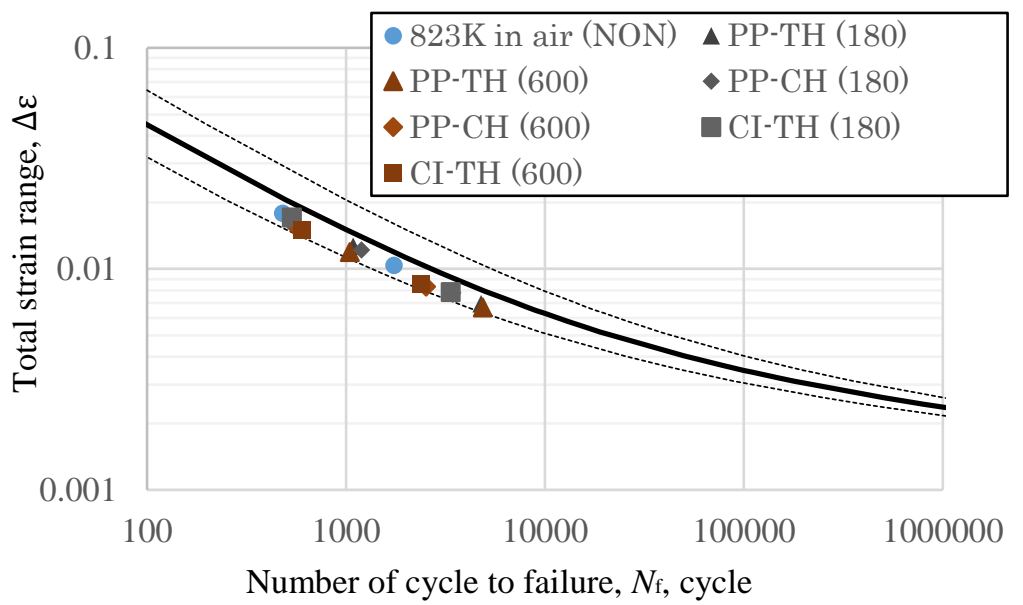

Fig. 6. Correlated $N_{\mathrm{f}}$ with Eq. (3).

\section{Discussion}

In the cause of the success in correlation using Eq. (3), the modified universal slope method and the conventional slope method (Eq. (1)) under push-pull were compared.

\subsection{Comparison of modified universal slope method to usual slope method}

Validity of the method of modified universal slopes was investigated by comparing with the method of conventional universal slopes. A strain range based on the method of universal slopes can be defined as,

$$
\Delta \varepsilon=\frac{\sigma_{\mathrm{B}}}{E} N_{\mathrm{f}}^{-0.12}+\varepsilon_{\mathrm{f}}^{0.6} N_{\mathrm{f}}^{-0.6}
$$

where $\sigma_{\mathrm{B}}, E$ and $\varepsilon_{\mathrm{f}}$ are the same value as the results in section 3.1.

Figure 7 shows correlated $N_{\mathrm{f}}$ using Eq. (1) at $823 \mathrm{~K}$ in air (Fig. (a)) and correlated $N_{\mathrm{f}}$ using Eq. (7) (Fig. (b)). Data plots were obtained from the push-pull loading at $823 \mathrm{~K}$ in air. The solid line on Figure7 shows the fatigue life curve based on the method of modified universal slopes and based on the method of universal slopes, respectively. In the result of Fig. (a), it can be confirmed that the fatigue data under push-pull loading at $823 \mathrm{~K}$ in air was evaluated within the factor of 2 band. On the contrary, as focusing on low total strain range region, those fatigue data on Fig. (b) was not evaluated within the factor of 2 band. Therefore, it can be confirmed that an analysis by the method of modified universal slopes has higher reliability than the analysis by the method of universal slopes. 


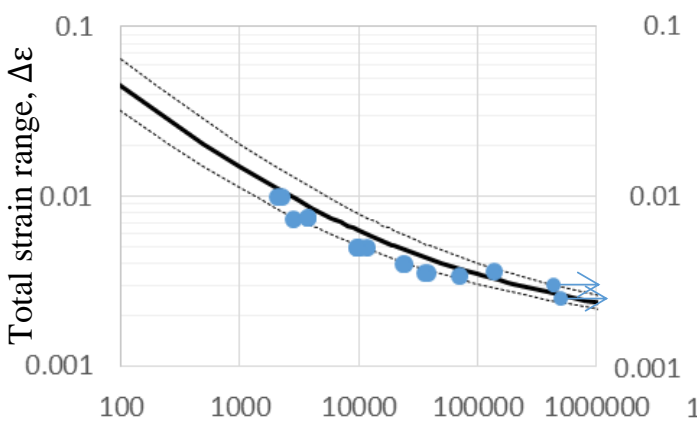

Number of cycle to failure, Nf, cycle

(a) Correlated $N_{\text {f }}$ with Eq. (1).

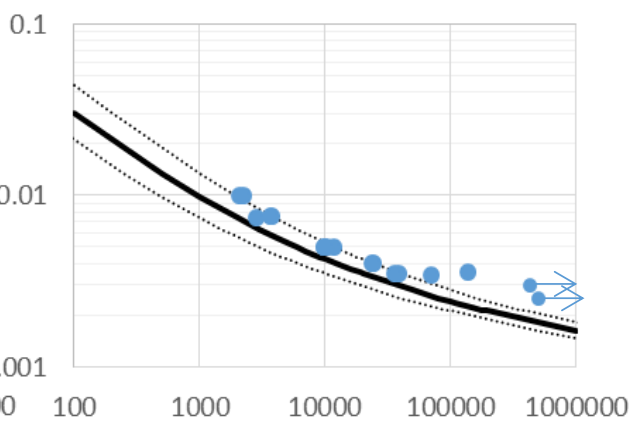

Number of cycle to failure, Nf, cycle

(b) Correlated $N_{\mathrm{f}}$ with Eq. (7).

Fig. 7. Comparison Eq. (1) with Eq. (7).

Moreover, the cause for the decrease was investigated by dividing total strain range into elastic and plastic portion of each method. Figure 8 shows the result of the method of modified universal slopes (Fig. (a)) and the method of universal slopes (Fig. (b)). The black line is each fatigue curve subjected to Fig. (a) and Fig. (b). Blue line and Orange line are elastic and plastic section of each equation, respectively. When comparing Fig. (a) with Fig. (b), it can be conformed that elastic strain is constant even though number of cycle to failure change. However, as focusing on plastic section, the value of that has changed dramatically in accordance with the change of number of cycle to failure. Moreover, the almost double difference was confirmed in low total strain range. Therefore, it can be confirmed that the data of F82H steel under push-pull loading at $823 \mathrm{~K}$ in air was evaluated within the factor of 2 band more precisely by means of the method of modified universal slopes. For the future study, collection of the fatigue data in low total strain range is necessary to investigate whether those data can be evaluated within the factor of 2 band or not.

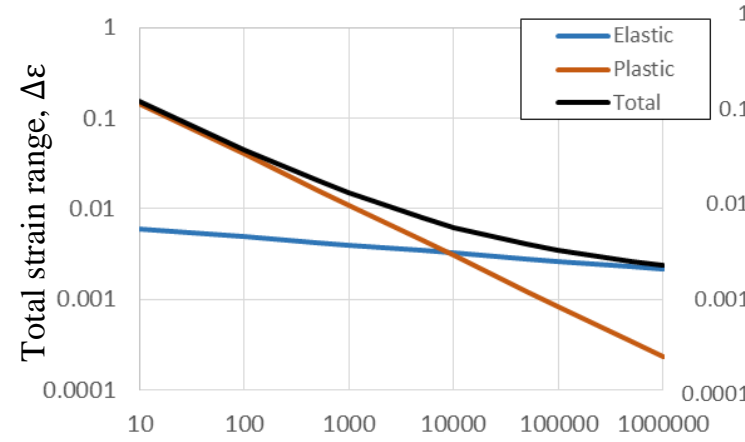

Number of cycle to failure, $\mathrm{Nf}$, cycle

(a) Correlation of $N_{\mathrm{f}}$ with Eq. (1).

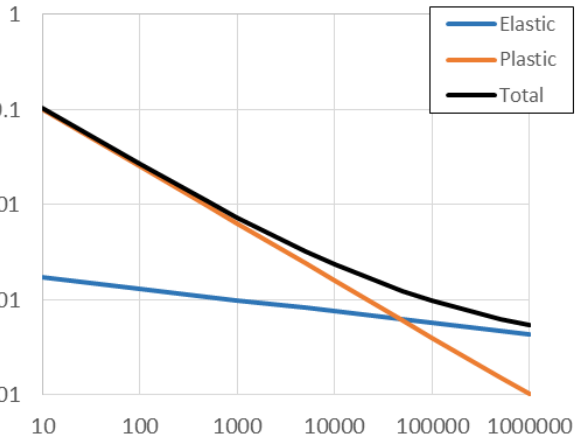

Number of cycle to failure, Nf, cycle

(b) Correlation of $N_{\mathrm{f}}$ with Eq. (7).

Fig.8. Comparison elastic strain with plastic strain. 


\section{Conclusions}

1. It is confirmed that the fatigue test data under push-pull loading can be evaluated properly by means of the method of modified universal slopes which uses static tensile properties.

2. The fatigue lives in room temperature and $823 \mathrm{~K}$ for $\mathrm{F} 82 \mathrm{H}$ steel are compatible.

3. The creep-fatigue life is evaluated within the factor of 2 band by means of combination of modified universal slope method and IS method which considers influences of both non-proportionality and creep damage.

\section{References}

1. E. Uematsu, T. Takeda and S. Nishio, JPS Conference Proceeding, 6 (2001).

2. S. Jitsukawa, A. Kimura and A. Kohyama, Journal of Nuclear Materials, 39-46 (2004).

3. K. Shiba, R. Kasada, S. Nogami, T. Nakata and N. Okubo, J. Plasma Fusion Res., 3 (2011).

4. T. Chida, M. Ida, H. Nakanura, T. Sato and M. Sugimoto, JAEA-Technology (2007).

5. T. Itoh, T. Yang, Journal of solid mechanics and materials engineering, Vol. 4, No. 10 (2010). 